\title{
Méthodes de calculs des potentiels et productibles associés aux énergies marines : études de cas en Manche-Atlantique
}

\author{
Sébastien LEDOUX, David CHOTARD, Vincent MAZEIRAUD, Nicolas GARCIA, Thibault SAILLARD, \\ Yvon MENSENCAL ${ }^{1}$, Hakim MOUSLIM ${ }^{2}$
}

1. ARTELIA Eau \& Environnement e-mail: sebastien.ledoux@arteliagroup.com ;

2. INNOSEA, e-mail : hakim.mouslim@innosea.fr

\begin{abstract}
RÉSUMÉ. - Face au développement des EMR (Energies Marines Renouvelables) ces dernières années et à la demande de collectivités publiques territoriales d'évaluer le potentiel énergétique de leur domaine côtier, il était nécessaire de se doter d'un outil intégré permettant de déterminer à l'échelle d'un site puis d'un territoire d'abord la ressource brute par type d'énergie, puis successivement le potentiel technique et technico-économique.

Cela a conduit les équipes d'ARTELIA à mobiliser leurs spécialistes en hydraulique maritime et fluviale et les énergéticiens pour développer un outil dédié au calcul des productibles EMR.

Dans cet objectif opérationnel, ARTELIA a mis en ouvre des actions R\&D afin de disposer de l'état de l'art des méthodes de calculs en vigueur et des outils existants et mis en œuvre dans les autres pays européens pionniers du domaine et en particulier au Royaume-Uni (Atlas of UK Marine Renewables Energy Ressources, ABP Mer,) et aux Etats- Unis (travaux de l'EPRI -Electric Power Research Institute- notamment).

L'outil a ensuite été développé et mis en œuvre successivement dans le cadre du calcul du potentiel EMR du domaine côtier de la Région Poitou-Charentes (Maître d'Ouvrage : Région Poitou-Charentes), puis à travers les missions d'études réalisées sur le potentiel hydrolien de Basse-Normandie (Maître d'Ouvrage : DREAL Basse-Normandie) et sur le potentiel EMR du littoral Aquitaine (Maître d'Ouvrage : Région Aquitaine - GIP Littoral Aquitain). L'outil permet successivement d'évaluer les gisements, potentiels techniques et technico-économiques des ressources EMR. Il a été appliqué à des études en lien avec des projets de fermes d'hydroliennes (offshore, en estuaires et rivières), houlomotrices (offshore, en zones littorale et côtière) et champs d'éoliennes offshore.
\end{abstract}

Mots-clés : énergies marines renouvelables, potentiel énergétique, productible énergétique

\section{Methods for calculations of potential and producible associated with marine energies: case studies in the Channel - Atlantic}

\begin{abstract}
Faced with the development of Marine Renewable Energy (MRE) in recent years and demand from regional public authorities to evaluate the energy potential of their coastal domains, it was necessary to design an integrated tool for determining, at the scale of a site and then a region, first the gross resource per energy type and then its technical potential followed by its technico-economic potential.

In response to this need, Artelia mobilised its experts in maritime and river hydraulics and in energy with the aim of developing a tool dedicated to calculating MRE production capacities.

With this operational objective in mind, ARTELIA undertook R\&D actions in order to determine the state of the art in calculation methods and in tools already developed and in use in other European countries spearheading this activity, especially the United Kingdom (Atlas of UK Marine Renewable Energy Resources, ABPmer,) and the United States (in particular the work of the EPRI (Electric Power Research Institute)).

The tool was then developed and applied successively in the framework of calculating the MRE potential of the coastal domain of the Poitou-Charentes region (client: Poitou-Charentes regional council), then through study assessments performed on the marine current power potential of Lower Normandy (client: DREAL Basse-Normandie) and on the MRE potential of the Aquitaine coast (client: Aquitaine regional council - GIP Littoral Aquitain). The tool allows for the assessment of the resources, technical and techno-economic potentials It has been applied to the following topics: marine current power (offshore and in estuaries and rivers), wave power (offshore, nearshore and coastal) and wind power (offshore and floating turbines).

This article provides a brief summary of the various aspects of the tool implemented, illustrated through a few examples drawn from the studies referred to above.
\end{abstract}

Key-words: marine renewable energy, potential, producible power 


\section{INTRODUCTION - OBJECTIFS DE L'ÉTUDE}

Un domaine émergeant mais qui demeure encore peu connu en termes de potentialités énergétiques est celui des " énergies marines renouvelables (EMR) », qu'elles soient issues par exemple de la marée, des courants, du vent, de la houle etc ... La France métropolitaine dispose d'un fort potentiel de développement pour les énergies renouvelables de la mer avec plus $4700 \mathrm{~km}$ de côtes et des zones maritimes de l'ordre de 11 millions de $\mathrm{km}^{2}$. De nombreux acteurs économiques et institutionnels présents sur le territoire national possèdent les compétences et l'expertise pour contribuer au développement de ces ressources marines.

Dans le cadre du déploiement EMR à venir, plusieurs régions françaises souhaitent profiter des potentialités offertes par leur domaine côtier pour jouer un rôle majeur dans le développement de la filière et les retombées économiques associées. Dans ce cadre, plusieurs collectivités territoriales ou services déconcentrés de l'Etat ont souhaité connaître la ressource EMR sur leur territoire ainsi que les contraintes et enjeux à prendre en compte dans un objectif de détermination des potentiels de développement EMR et des productibles associés. Ainsi, la Région Poitou-Charentes en 2010, la DREAL Basse-Normandie en 2010-2011 puis la Région Aquitaine et le GIP Littoral Aquitain en 2011-2012 ont successivement confiées aux équipes de SOGREAH, devenu ARTELIA Eau \& Environnement, des études sur les potentialités EMR de leurs « territoires côtiers ».

En complément des approches déjà utilisées par le CETMEF dans le cadre de la planification Eolien offshore puis EMR à l'échelle de la façade Manche - Mer du Nord et Atlantique, ARTELIA a pu proposer des méthodologies détaillées et adaptées aux caractéristiques et ressources propres des territoires concernés. En effet, la combinaison des savoir-faire et des compétences des équipes dans le domaine de l'hydraulique maritime et fluviale, de la modélisation numérique complexe et dans le domaine de l'énergie a permis pour chacune de ces études :

- D'identifier à partir d'états de l'art détaillés, les technologies potentielles de conversion d'énergie (hydrolien, houlomoteur, marémoteur, etc ...) et leur adaptabilité aux territoires d'étude concernés,

- De déterminer les zones d'accueil potentielles sur la base de seuils définis par le CETMEF ou par la littérature scientifique et du recensement et de la cartographie SIG détaillée des contraintes, enjeux environnementaux et usages maritimes, côtiers ou estuariens,

- De réaliser dans un premier temps des calculs du gisement énergétique qui représente les caractéristiques de la ressource. Ce calcul provient du croisement des résultats issus de modélisations des courants et des houles sur les zones d'étude et des contraintes et enjeux sur les territoires, - De faire ensuite des calculs des potentiels techniques et technico-économiques.

- De définir sur la base des éléments précédents des scénarios de déploiement des EMR à différentes échelles de temps sur les territoires concernés.

Ces études pluridisciplinaires ont nécessité le travail conjoint de différents spécialistes d'ARTELIA dans les domaines de l'ingénierie maritime et côtière, de l'environnement littoral, de la modélisation numérique, de l'énergie et électricité, et des actions de R\&D internes pour le développement des outils. Un appui scientifique a par ailleurs été apporté par un expert d'INNOSEA, co-auteur de cet article.
A travers ces différentes études menées depuis 2010, les équipes d'ARTELIA ont pu mettre en œuvre, fiabiliser et optimiser les méthodes d'analyse et de calculs, développer des outils numériques dédiés sur les sujets EMR suivants :

- Potentiel houlomoteur offshore, nearshore et côtier en particulier sur la côte Aquitaine et en Charente-Maritime,

- Potentiel hydrolien fluvial de petite capacité dans les estuaires et Pertuis Charentais, estuaires de la Gironde, Bassin d'Arcachon et Adour,

- Potentiel hydrolien de grande puissance en BasseNormandie en particulier sur les sites du Raz Blanchard et du Raz de Barfleur,

- Potentiel Eolien posé et flottant (littoral Aquitain).

Ces méthodologies d'analyse et de calcul pour la détermination du potentiel énergétique d'un site puis à l'échelle d'un territoire sont présentées ci-après à partir d'exemples et d'illustrations issues des études menées sur les domaines côtiers régionaux étudiés.

\section{DETERMINATION DU POTENTIEL ENERGETIQUE - PRINCIPE GENERAL}

Le principe général de la méthodologie de calcul est d'évaluer le potentiel technico-économique par les étapes suivantes :

- Calcul du gisement énergétique qui représente les caractéristiques de la ressource, sans prise en compte des dispositifs technologiques de récupération de l'énergie. Ce calcul est réalisé à partir des analyses sur le gisement,

- Calcul du potentiel technique qui est lié aux caractéristiques des équipements de récupération de l'énergie et du raccordement à terre envisagés (chutes de tension, pertes du transformateur, disponibilité annuelle du réseau électrique, puissance maximale de raccordement d'un parc). Ce calcul est indépendant de toutes considérations économiques,

- Calcul du potentiel technico-économique qui introduit un chiffrage du potentiel et vise à minimiser le coût pour la collectivité du $\mathrm{kWh}$ produit tout en assurant une rentabilité économique suffisante pour l'investisseur. Ce potentiel dépend des deux paramètres suivants : le coût maximum de l'énergie produite et la dimension maximale d'un parc. Le coût de l'énergie produite doit être comparé avec le tarif d'achat de l'électricité et, pour que le projet soit effectivement intéressant pour un investisseur, il est nécessaire qu'il dégage une marge.

Ce principe général est représenté sur la figure 1 .

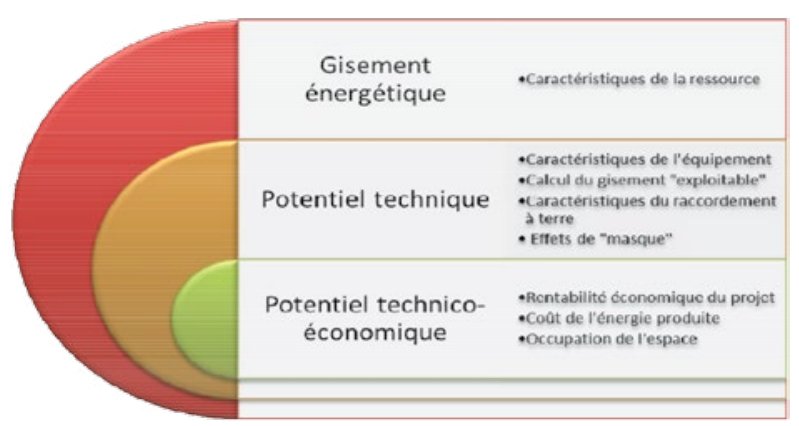

Figure 1 : Principe méthodologique de calcul du potentiel technico-économique. 


\section{POTENTIEL ENERGETIQUE D'UN SITE}

\section{III.1. Gisement énergétique}

Dans le cadre de la planification nationale relative aux énergies marines, le CETMEF a déterminé des seuils et critères techniques reposant à la fois sur une notion de bathymétrie et de vitesse de vent (éolien), de vitesse de courant (hydrolien) ou de puissance de la houle incidente (houlomoteur) (http://www.geolittoral.developpement-durable.gouv.fr/la-planification-a273.html). Selon la nature de la technologie considérée et les caractéristiques du site ou du domaine d'étude, ces critères techniques ne sont pas totalement adaptés. Ils doivent ainsi parfois être précisés sur la base de littérature scientifique et technique sur le sujet. Des modélisations numériques ont été mises en œuvres pour évaluer ces gisements. Elles sont décrites sommairement ci-après (nota : le gisement éolien n'a pas été traité de manière détaillé dans ces études. Il n'est donc pas présenté ici).

\section{III.1.1. Calcul du gisement hydrolien}

Le contenu énergétique de la ressource hydrolienne à un instant $t$ donné dépend de la vitesse du courant et de la hauteur de la colonne d'eau. Le gisement hydrolien est déterminé à l'aide d'un modèle hydrodynamique bâti à l'aide du code TELEMAC développé par EDF-DER ${ }^{1}$. L'exploitation des résultats du modèle hydrodynamique $2 \mathrm{D}$ ou $3 \mathrm{D}$ selon le degré de précisions attendu permet de disposer des paramètres utiles à la caractérisation de la ressource (marnage

1. DER : Direction Etudes et Recherche

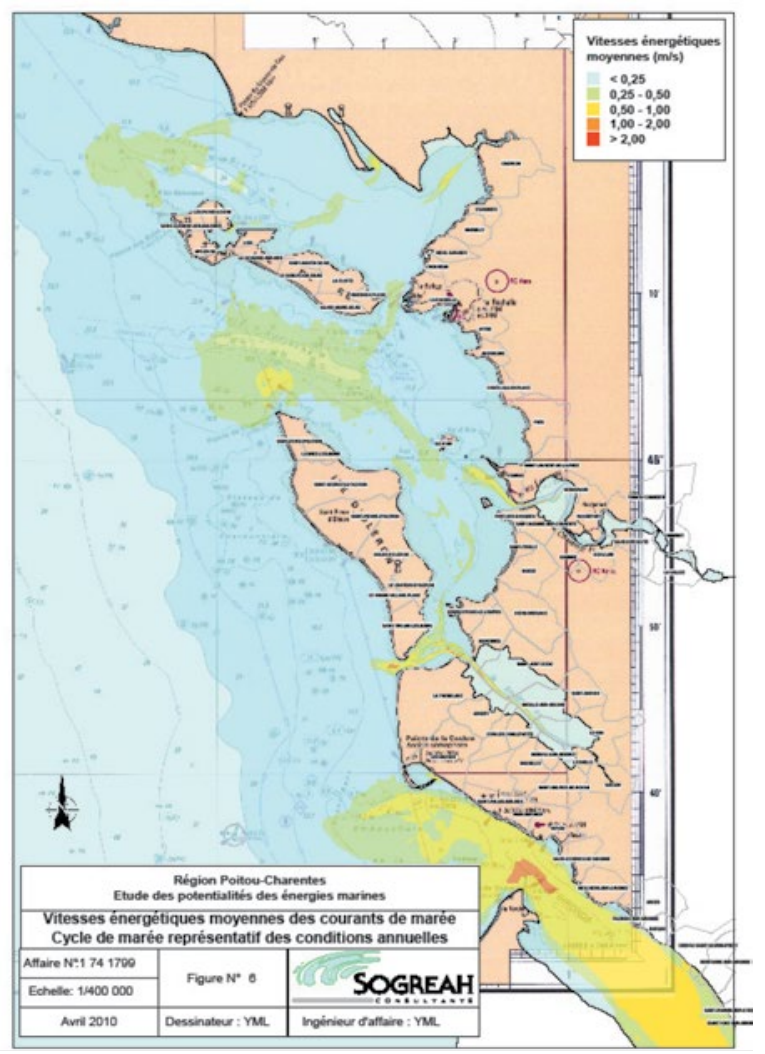

Figure 2 : Gisement hydrolien Poitou-Charentes.

Source : SOGREAH, 2010 maximal, vitesses instantanées maximales, puissance maximale instantanée, énergie moyenne annuelle).

\section{III.1.2. Le potentiel houlomoteur}

Le potentiel énergétique du aux vagues est habituellement caractérisé et quantifié à l'aide du flux linéique de puissance des vagues (i.e. flux de puissance par unité de largeur de crête de vague en $\mathrm{kW} / \mathrm{m}$ ). L'évaluation du gisement houlomoteur s'appuie sur le logiciel TOMAWAC (chaine TELEMAC) de propagation des vagues, alimenté par des données de type ANEMOC (Atlas Numérique d'Etats de Mer Océanique et Côtier développée conjointement par EDF R\&D LNHE et le CETMEF) ou autre bases si disponibles (par exemple la base de données BOBWA-H (Bay of Biscay Wave Atlas - Hindcast.- http://bobwa.brgm.fr/ pour le littoral aquitain). La détermination du potentiel de houle nécessite des bases de données d'états de mer relativement précises et sur des périodes suffisamment longues pour être représentatives d'un point de vue statistique.

La recombinaison des conditions de houles disponibles à la frontière du modèle TOMAWAC est ensuite réalisée sur la période statistique considérée. Cette méthode permet de calculer en chacun des points du modèle mis en œuvre les conditions de houle, avec un pas de temps horaire (hauteur, direction et période). Ceci permet ensuite de déterminer les puissances instantanées associées à la houle et l'énergie incidente à l'échelle d'une année type (moyenne sur les années disponibles). A titre d'illustration, les figures ci-dessous représentent le gisement hydrolien calculé sur le littoral de la région Poitou-Charentes (figure de gauche) et le gisement houlomoteur aquitain (figure de droite).

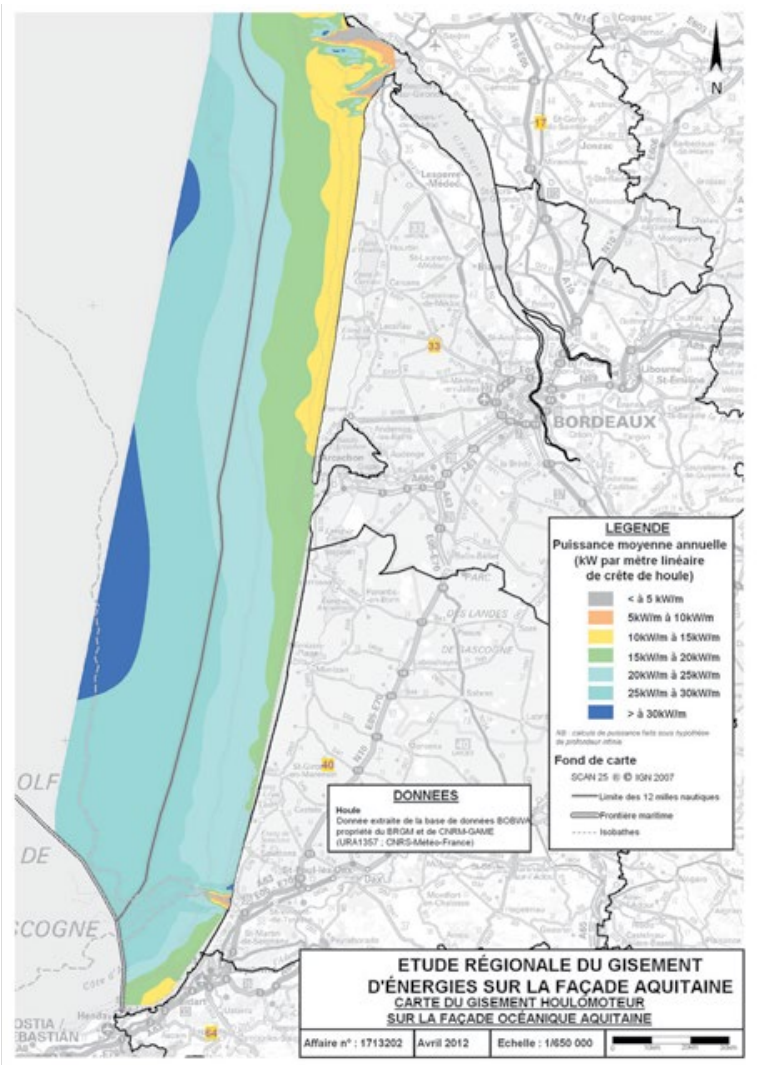

Figure 3 : Gisement houlomoteur Aquitain.

Source : ARTELIA, 2012 


\section{III.2. Potentiel technique d'une installation}

Le potentiel technique pour une longueur de front d'énergie donnée (vent, courant, houle) est défini par l'énergie électrique injectée sur le réseau par un parc dont la longueur des rangées est celle du front d'énergie, et sans limitation du nombre de rangées. Ce potentiel n'est donc pas économiquement réaliste mais donne un plafond dans l'état actuel des technologies hors considérations économiques. Le calcul du potentiel technique nécessite de disposer de plusieurs types d'informations; décrits ci-après.

- Les caractéristiques des équipements de référence

A partir de l'analyse de l'adaptabilité des technologies aux territoires étudiés, des équipements de référence sont retenus pour l'exploitation de chaque ressource. Sur un même territoire, il est possible - et probable - que les caractéristiques de l'équipement de référence varient selon la zone considérée et le niveau de ressource. Par exemple, les caractéristiques utilisées pour modéliser l'équipement de référence hydrolien sont les suivantes : hauteur, largeur, vitesse nominale de fonctionnement, vitesse de mise en sécurité, vitesse de démarrage, direction normale de l'hydrolienne, coefficient de puissance, durée de vie - sur laquelle l'ensemble des calculs économiques sont réalisés -, disponibilité annuelle, rendement de transmission mécanique, rendement de la génératrice etc ...

Les caractéristiques utilisées pour modéliser l'équipement de référence houlomoteur sont les suivantes : matrice de puissance (les matrices de puissance utilisées dans le modèle sont issues de compilations et extrapolations de matrices de puissance obtenues par modélisation ou mesures de technologies en développement), puissance électrique nominale, angles d'incidence, durée de vie, disponibilité annuelle, rendement de transmission mécanique, rendement de la génératrice etc ...

- Le calcul du gisement dit « exploitable »

Le gisement exploitable correspond à la part du gisement qu'il est possible d'exploiter par une machine donnée compte tenu de ses caractéristiques, comme par exemple les seuils de vitesses de démarrage (faibles vitesses) ou de mise en sécurité/arrêt (fortes vitesses).

- Les caractéristiques du raccordement électrique

L'impact du raccordement au réseau électrique sur le productible est modélisé par les paramètres suivants : chute de tension entre le point de raccordement et la sortie des machines, pertes du transformateur, disponibilité annuelle $\mathrm{du}$ réseau électrique, puissance maximale de raccordement d'un parc.

- La prise en compte des effets de "masque » d'une rangée de machines à l'autre et d'un parc à l'autre dans l'outil développé par ARTELIA.

Dans le cas de plusieurs rangées successives de machines, le calcul du productible d'une rangée donnée doit prendre en compte les modifications des caractéristiques de la ressource induites par la rangée précédente. L'état de la ressource (hauteur significative et période de pic pour la houle et vitesse pour le courant) en aval d'une rangée de machine est calculé à partir d'un bilan en énergie sur le principe de conservation de l'énergie : l'énergie résiduelle à l'aval d'une rangée est égale à l'énergie incidente moins l'énergie absorbée par les machines. La figure 4 illustre par exemple l'évolution du productible en fonction du nombre de rangées d'un parc houlomoteur sur un des sites étudié du littoral aquitain. Le potentiel technique est atteint au niveau du plafonnement du productible, potentiel n'ayant pas de sens économique.

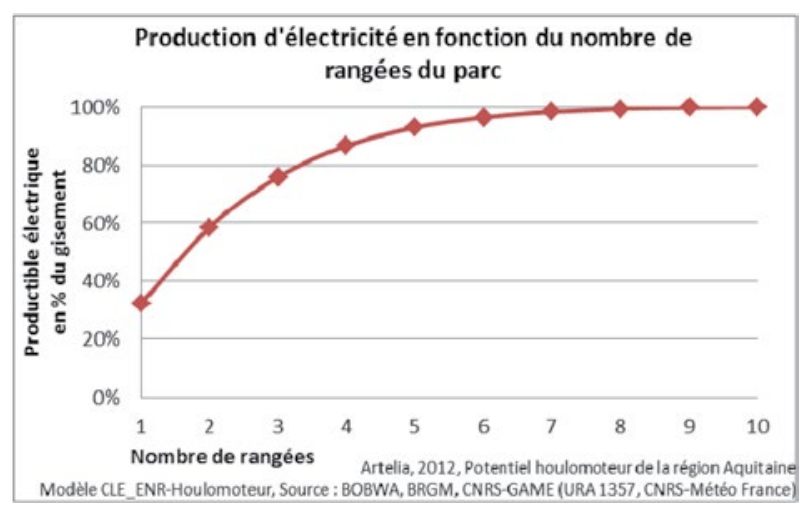

Figure 4 : Production d'électricité en fonction du nombre de rangées du parc. ARTELIA, 2012

\section{III.3. Potentiel technico-économique d'un site}

Afin de déterminer un potentiel ayant plus de sens auprès des décideurs politiques ou bien d'investisseurs potentiels, il est nécessaire d'introduire des éléments économiques dans l'évaluation du potentiel.

\section{III.3.1. Calcul économique d'une installation}

Etant donné le niveau de maturité de la plupart des technologies EMR qui sont pour les plus avancées à un stade prototype ou pré-industriel, les données économiques sont à considérer avec une incertitude significative. Pour cela, l'ensemble des calculs économiques sont menés avec une fourchette de valeurs dites " optimistes » et «pessimistes». Les coûts considérés sont les suivants :

- coût d'investissement : études, convertisseurs et équipements électriques, installations, raccordement en mer, raccordement sur terre,

- coûts annuels de fonctionnement/maintenance,

- conditions économiques de développement : taux d'actualisation, tarif d'achat.

A partir de ces éléments, le coût de l'énergie produite par un parc donné peut être évalué et doit être comparé avec le tarif d'achat de l'électricité. L'indicateur retenu pour évaluer l'attractivité du projet pour un investisseur est un indice de profitabilité, ratio entre la valeur actuelle nette d'une opération et son coût d'investissement initial, indicateur calculé selon la Méthode du Taux d'Enrichissement en Capital de Bernard Chabot (ADEME, 2002) et tenant compte de l'ensemble des frais inhérents au développement d'un site : des études amont à son installation et maintenance, jusqu'au démantèlement, ainsi que la prise en compte du coût de l'accès au capital. Selon Bernard Chabot, cet indice de profitabilité (IP) doit être supérieur ou égal 0,3 pour que le projet soit attractif pour un investisseur. Si ce seuil n'est pas atteint, un soutien financier complémentaire est alors nécessaire. Celui-ci peut prendre de multiples formes comme par exemple une augmentation du tarif d'achat ou le financement d'une partie de l'investissement. Ces deux formes de soutien ont un impact différent sur l'économie du projet, toutefois, pour la suite du présent travail, le paramètre utilisé est le tarif d'achat de l'énergie produite.

\section{III.3.2. Dimensionnement d'un parc à l'optimum technico-économique}

Les dimensions du parc ont un impact direct sur l'indice de profitabilité par effet d'échelle sur les coûts fixes. 
L'évolution de l'indice de profitabilité en fonction du nombre de rangées permet de mettre en évidence la taille du parc à partir de laquelle l'augmentation du productible n'est économiquement pas intéressante. La figure 5 (gauche) illustre ce point dans un cas où le parc n'est jamais économiquement acceptable pour un développeur de projet $($ IP $<0,3)$. Dans une telle situation, il est nécessaire de modifier les conditions économiques de développement du parc. Le modèle calcule alors le soutien financier minimum à apporter au projet pour que le sommet de la courbe de l'évolution de l'indice de profitabilité corresponde à un IP $=0,3$. Dans l'exemple décrit ici (figure de droite), l'optimum technico-économique sur cette zone est obtenu pour 5 rangées.

Le potentiel technico-économique est donc fonction du coût de l'énergie maximum que la collectivité consent à payer. Notons que les premiers parcs seront probablement conçus de petite taille avec un nombre réduit de machines par rangée, puis, la maîtrise des risques s'améliorant et le niveau de maturité des filières concernées se développant, les parcs pourront voir leurs dimensions sensiblement augmenter.

Afin de mieux appréhender l'impact de la taille d'un parc sur le potentiel technico-économique d'une zone en fonction du coût de l'énergie que la collectivité est prête à payer, l'approche de calcul suivante, appliquée sur chaque zone de calcul, a été retenue dans l'outil présenté ici :

- Variable : nombre de machines par rangée, correspondant à la longueur du front de houle incident sur le parc, l'espacement des machines étant une caractéristique fixe propre à la technologie.

- Pour chaque cas, calcul du nombre de rangées donnant l'optimum technico-économique : nombre de rangées permettant d'atteindre un indice de profitabilité (IP) de 0,3 pour un soutien financier minimum,

- Calcul de la puissance installée, du productible et du coût de l'énergie produite du parc ainsi dimensionné.

La figure 6 illustre l'évolution du potentiel technicoéconomique par unité de front de houle incidente d'un site en fonction du nombre de machines par rangée (indiqué par les nombres à côté des symboles), pour des parcs dont le nombre de rangées est calculé de façon à ce que le prix de vente de l'énergie soit le plus faible possible tout en maintenant un IP de 0,3. Lorsque le nombre de machines par rangée augmente, le coût de l'énergie produite décroit. Toutefois, sur cet exemple, à partir de 30 machines par rangée le potentiel technico-économique unitaire décroit aussi avec l'augmentation de la dimension d'une rangée : cela traduit le fait qu'en augmentant la puissance

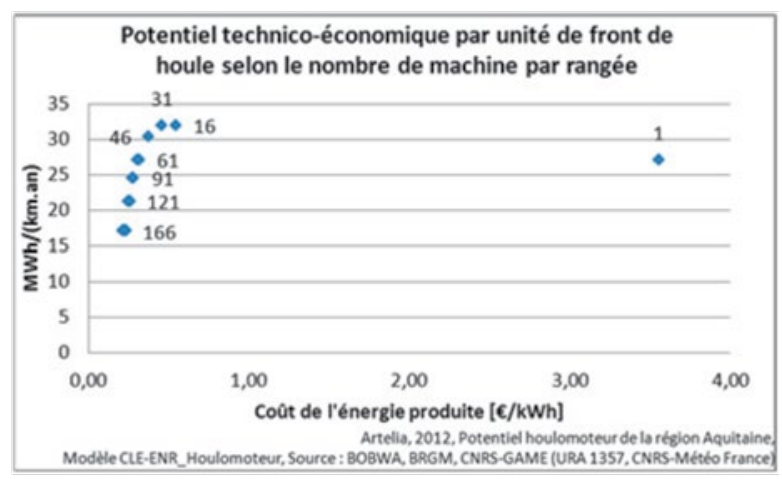

Figure 6 : Potentiel technico-économique de plusieurs zones potentielles ou parcs par unité de front de houle selon le nombre de machines par rangée. ARTELIA, 2012

installée par rangées, le nombre de rangées nécessaire pour atteindre un indice de profitabilité de 0,3 est plus faible. Or, avec moins de rangées, le parc extrait une part plus faible de l'énergie incidente.

\section{POTENTIEL ENERGETIQUE D'UN TERRITOIRE}

Le travail présenté dans la partie précédente est mené sur chaque nœud d'un maillage du territoire. Le maillage est construit de façon à ce que les caractéristiques de la ressource et du milieu soient homogènes sur une maille. Cette partie de l'article décrit la méthodologie suivie pour estimer le potentiel technico-économique d'un territoire à partir des potentiels technico-économiques de chaque maille.

\section{IV.1. Sélection d'un site}

Les sites pour lesquels le coût de l'énergie produite est trop important pour qu'ils puissent être raisonnablement valorisés sont exclus du bilan territorial. Pour cela, un seuil sur le coût de l'énergie produite est introduit. Ce seuil est défini par rapport aux coûts d'achat de l'électricité fixés par rapport aux projections du coût du kWh des technologies au stade industriel. Lorsque plusieurs mailles sont positionnées sur une même veine de courant ou le même front de houle incident, la somme des potentiels de ces mailles n'a pas de

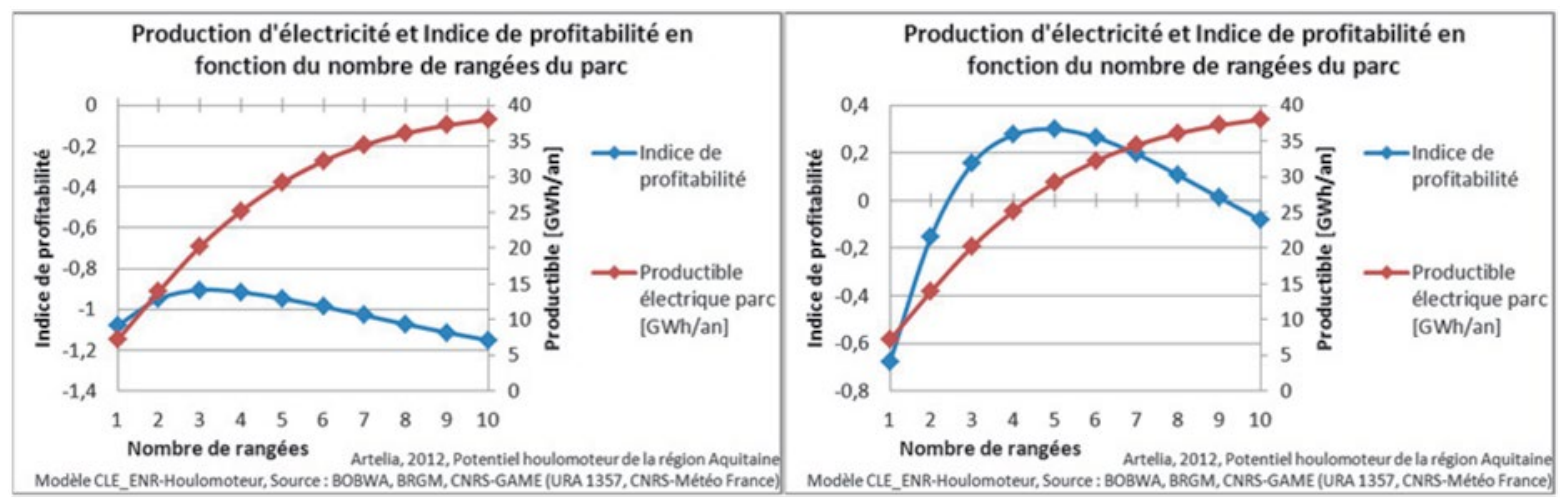

Figure 5 : Production d'électricité et indice de profitabilité en fonction du nombre de rangées du parc. ARTELIA, 2012 
sens car une partie de la ressource incidente sur la seconde maille a déjà été exploitée sur la première maille. La maille conservée est celle pour laquelle le coût de l'énergie produite est le plus faible.

\section{IV.2. Potentiel du territoire selon les caractéristiques du parc}

Le potentiel du territoire est alors la somme des potentiels des mailles ainsi sélectionnées. La figure 7 illustre le potentiel technico-économique houlomoteur offshore obtenu pour le littoral Aquitain. Chaque maille de calcul sur le territoire est notée Oxx dans le présent exemple. Le potentiel donné ici est un potentiel total à l'échelle de la maille, somme des productions de parcs juxtaposés valorisant l'ensemble du front de houle incident sur la maille. Cela fait apparaître que si seulement quelques mailles sont économiquement pertinentes pour de petites installations, qui pourront être privilégiées pour les projets démonstrateurs et pilotes, un potentiel significatif existe pour des parcs de grandes dimensions.

\section{OUTILS D'AIDE A LA DÉCISION}

\section{V.1. Cartographie des enjeux du territoire}

\section{V.1.1. Environnement et usages}

De manière générale, l'analyse de la faisabilité d'installations d'énergies marines doit s'appuyer sur un nombre important de données de base : contraintes océanographiques, environnementales, humaines ou encore réglementaires. C'est grâce à la juxtaposition de ces différentes données et des contraintes qu'elles peuvent représenter qu'il est possible d'aboutir à la détermination de zones potentielles d'implantation des parcs d'énergies marines. Il est à noter cependant que, dans le cadre de ce type d'étude, la synthèse des zonages est réalisée à une macro-échelle sur l'ensemble du domaine côtier et sur la base d'informations assez hétérogènes. Dans la méthodologie proposée, cette première approche permet cependant d'exclure un certain nombre de secteurs déjà qualifiés, soit sensibles soit non compatibles avec les projets de récupération d'énergies marines. Des analyses ultérieures plus fines des contraintes

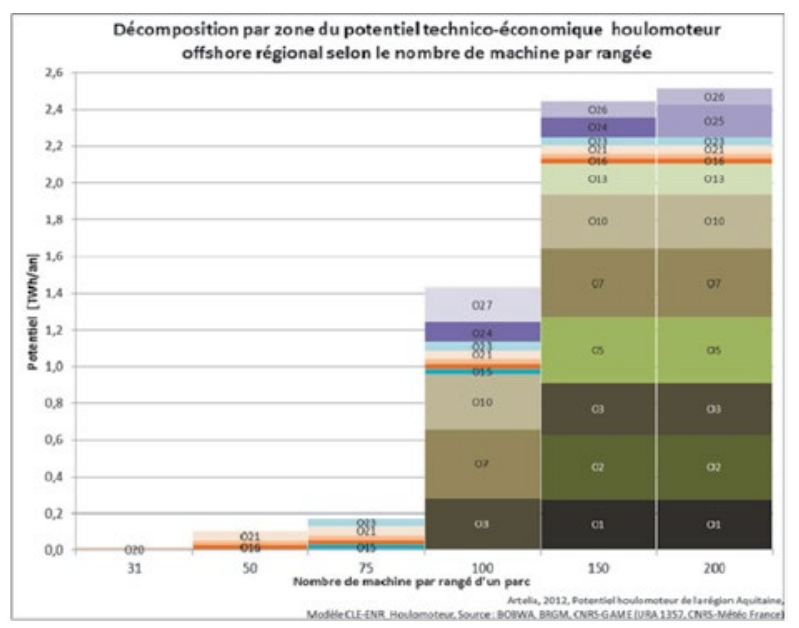

Figure 7 : Décomposition du potentiel technico-économique régional selon le nombre de machines par rangée.

ARTELIA, 2012 sont ensuite nécessaires pour les zones d'intérêts plus restreintes et/ou dans le cadre de l'évaluation des impacts des projets.

\section{V.1.2. Potentiel de production}

Un rendu cartographique du potentiel de production dans des conditions données (dimension du parc et coût maximum de l'énergie produite) et du coût de l'énergie produite associé à ce potentiel peut être réalisé, comme illustré sur la figure 8 dans le cas du littoral Aquitain.

\section{V.2. Comparaison potentiel de production/ coût de l'énergie}

En positionnant chaque maille de calcul sur un graphique donnant le potentiel technico-économique de la maille en fonction du coût de l'énergie (cf. figure 9), des points remarquables de la zone étudiée peuvent être identifiés. Ces données complètent les résultats cartographiques en faisant apparaître les sites sur lesquels un approfondissement de la faisabilité de telles installations serait pertinent à mener en priorité.

\section{V.3. Potentiel de production selon le coût de l'énergie et la taille du parc}

L'outil développé fournit au final le potentiel technicoéconomique du territoire concerné sur la base des implications économiques - coût de l'énergie produite - et de dimension des parcs. Il permet ainsi au décideur de définir sa stratégie à partir de ces deux paramètres. La figure 10 illustre le graphique obtenu avec le cas de l'houlomoteur offshore en région Aquitaine.

Ce graphique montre les iso-lignes de potentiel de production d'énergie selon le coût maximum de l'énergie produite et le nombre de machines par rangée. Dans cet exemple, pour des parcs de l'ordre de 100 machines par rangée, un potentiel de 5 TWh existe pour un coût de l'énergie inférieur à $0,22 € / \mathrm{kWh}$. En restreignant la largeur du parc à 50 machines par rangée, et en maintenant le seuil de coût de l'énergie produite à $0,22 € / \mathrm{kWh}$, le potentiel est réduit à $1 \mathrm{TWh}$. Enfin, la valorisation de la totalité du potentiel nécessiterait d'accepter des coûts de l'énergie produite allant jusqu'à $0,35 € / \mathrm{kWh}$, voire $0,44 € / \mathrm{kWh}$ pour quelques sites.

\section{CONCLUSIONS}

Afin de répondre aux attentes et besoins des collectivités publiques et industriels, le développement des Energies Marines Renouvelables (EMR) requiert la mise au point des méthodologies et outils d'approche des potentiels énergétiques et productibles associés. La méthodologie développée et les outils mis en œuvre par ARTELIA ces dernières années pour le compte de différents Maîtres d'Ouvrages, et améliorés au fur et à mesure des différentes études semblent répondre correctement aux besoins exprimés. Il convient cependant d'évoquer les limites inhérentes à ces méthodes et outils :

- Fiabilité des données d'entrée et leur validité pour le domaine étudié (mesures en mer, matrices de puissances des équipements ...) pouvant conduire à des incertitudes,

- Schématisation des processus et degré de précision lié à l'emprise des zones d'étude (des approches plus fines sont 


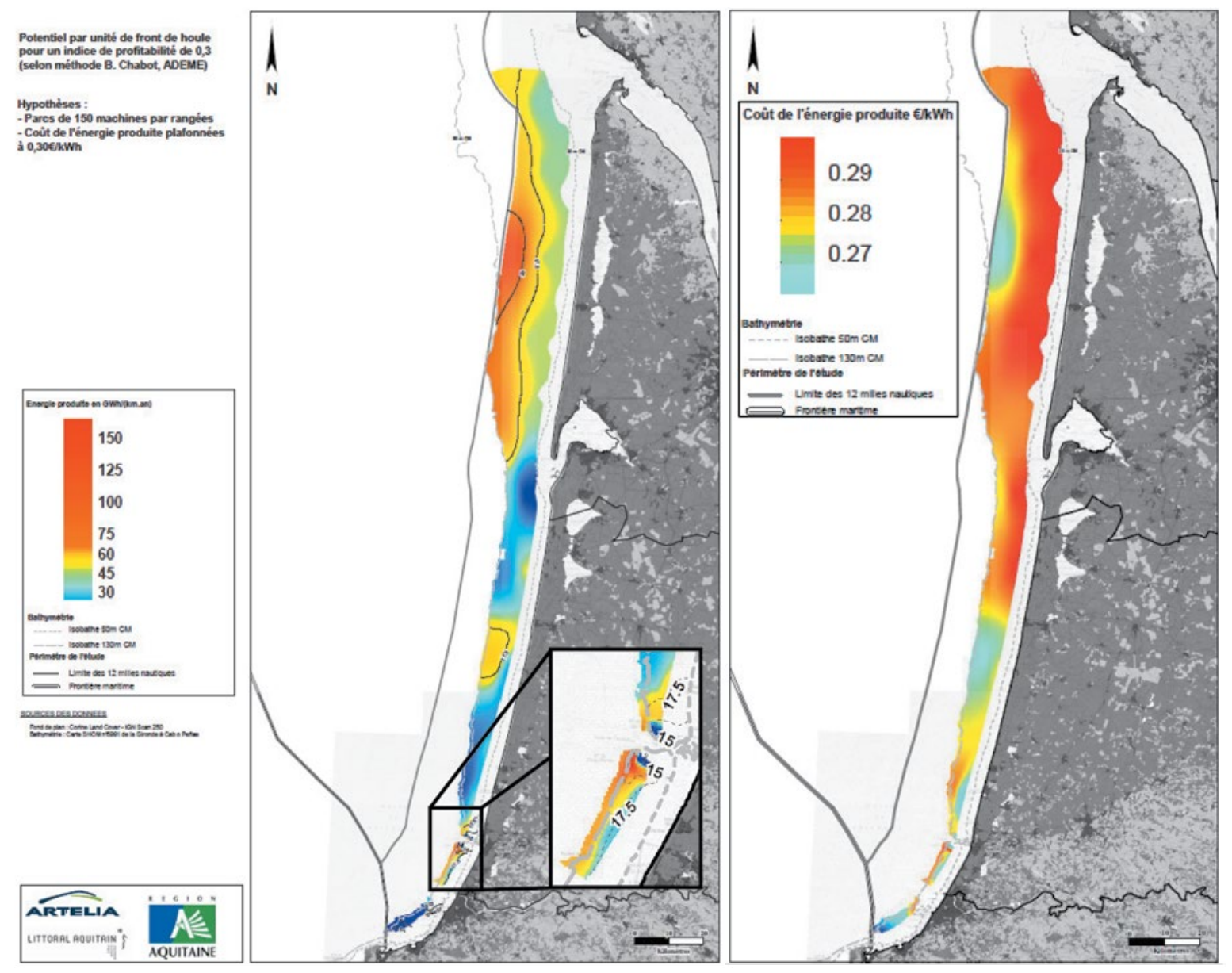

Figure 8 : Potentiel technico-économique houlomoteur nearshore littoral Aquitain. ARTELIA, 2012

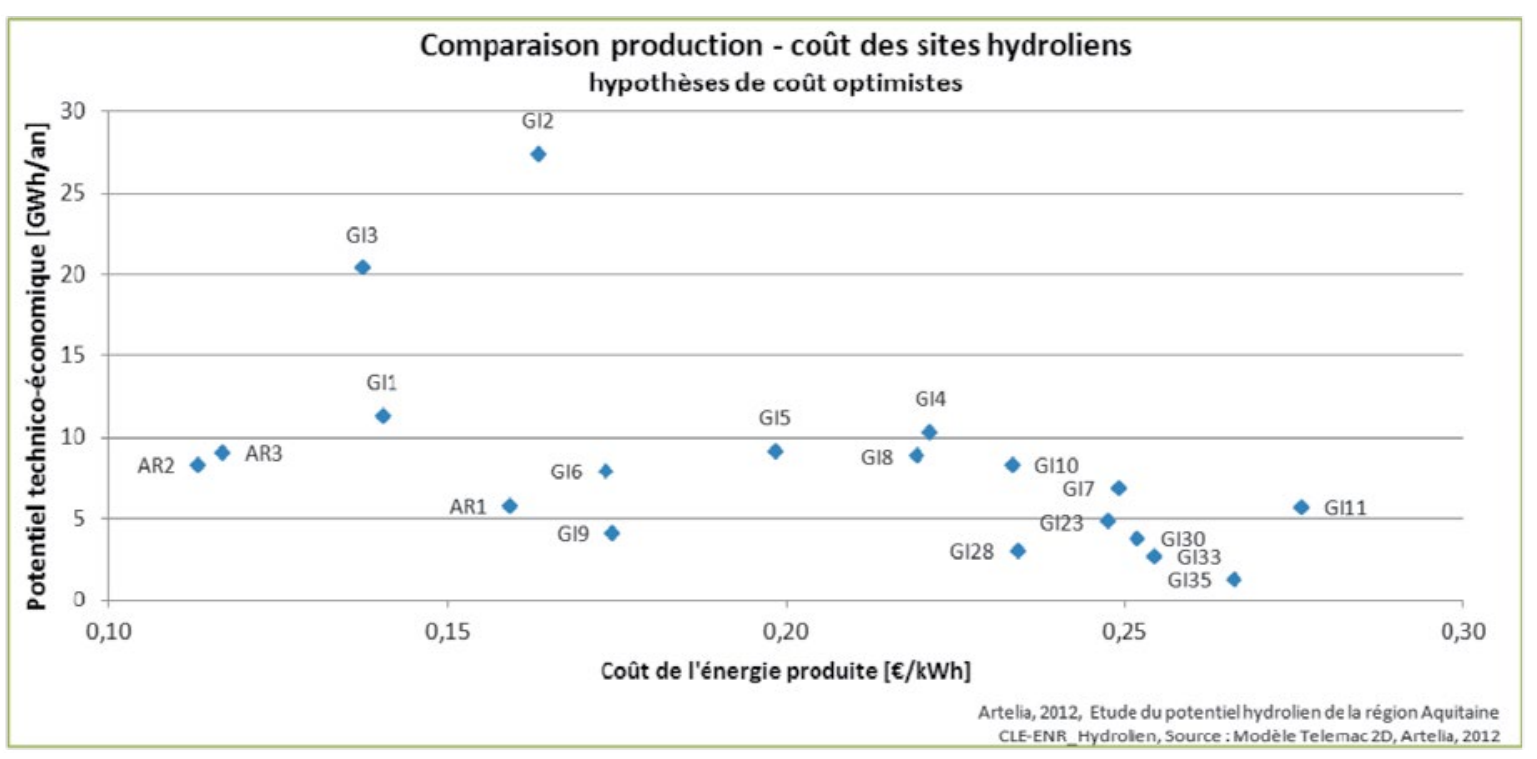

Figure 9 : Comparaison des sites selon leur potentiel et le coût de l'énergie associé. ARTELIA, 2012

mises en œuvre actuellement par ARTELIA dans le cadre d'études détaillées sur des sites en développement),

- Absence de retours d'expérience précisément renseignés dans la littérature scientifique sur des installations en fonctionnement, permettant une comparaison entre les approches de modélisation et les rendements effectifs.
Le travail d'amélioration et d'optimisation des outils est donc à poursuivre notamment à travers des programmes de R\&D collaboratifs tels que proposés dans le cadre de France Energies Marines (http://www.france-energies-marines.org/) ou EMACOP (http://www.emacop.fr/) en France et d'autres programmes à l'échelle européenne. 


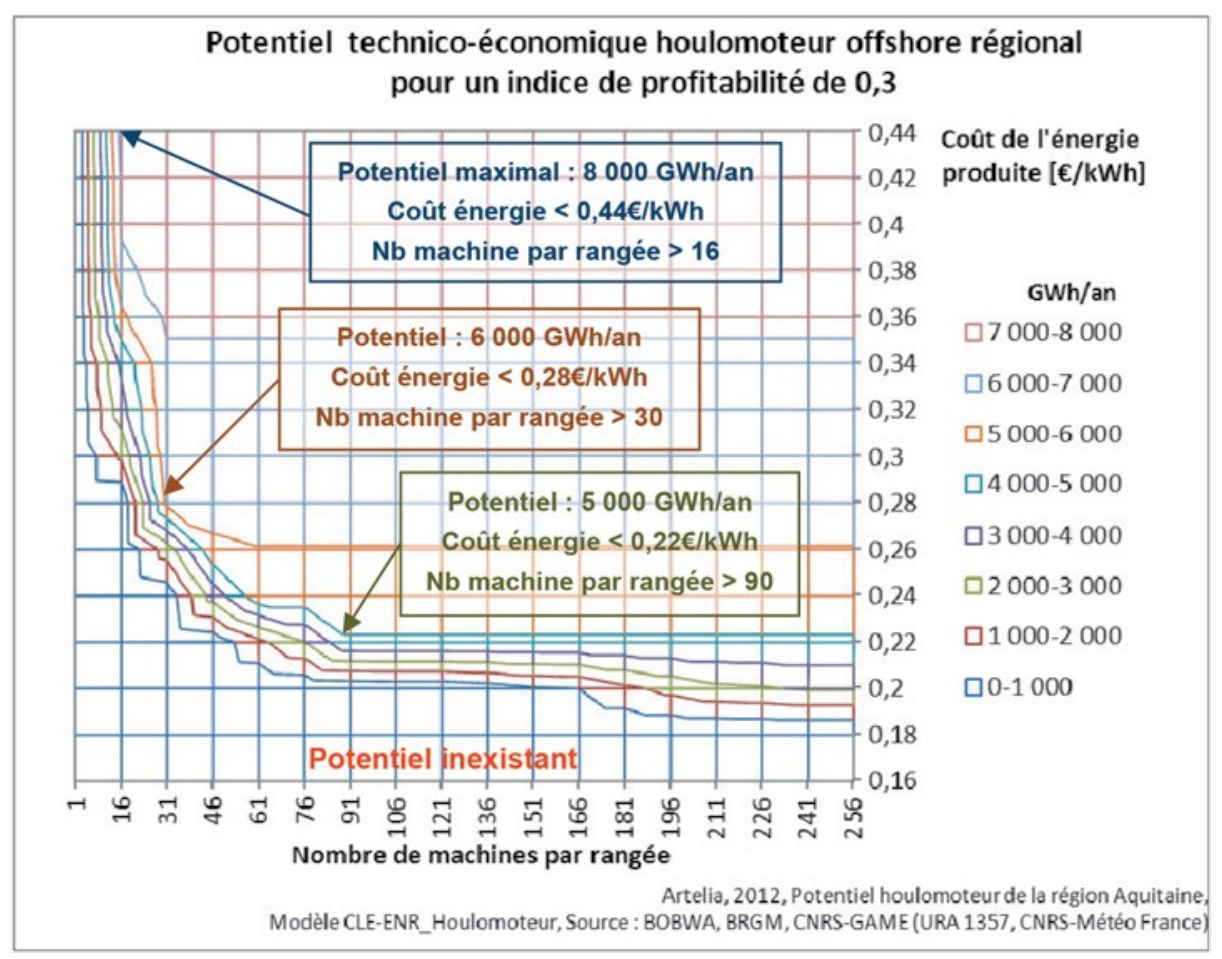

Figure 10 : Potentiel technico-économique houlomoteur offshore régional.

\section{REFERENCES}

G. Mattarolo, F. Lafon, M. Benoit (2009) - Wave energy ressource off the French Coasts : the ANEMOC database applied to the energy yield evaluation of Wave Energy Converters. Proceedings of the 8th European Wave and Tidal Energy Conference, Uppsala, Sweden.

M. Benoit, F. LAFON (2004) - A nearshore wave atlas along the coasts of France based on the numerical modelling of wave climate over 25 years. Proc. 29th Int. Conf. On Coastal Eng. (ICCE'2004), Lisbon (Portugal). 714-726

ARTELIA (2012) - Etude sur les potentialités en énergies marines en Aquitaine. Conseil Régional d'Aquitaine/GIP Littoral Aquitaine.

Bernard CHABOt (2002) — La méthode TEC d'analyse économique des projets énergétiques, ADEME.

SOGREAH (2010) — Etude des potentialités en énergies marines Poitou-Charentes, Conseil Régional Poitou-Charentes. 